\title{
Common iliac artery mycotic aneurysm due to melioidosis
}

\author{
Wedagedara VM, Nuwan C, Ubayasiri RA \\ Department of Vascular and Transplant Surgery, Teaching Hospital Karapitiya, Galle, Sri Lanka.
}

Correspondence: Dr. V. M. Wedagedara

e-mail: venurawedagedara@gmail.com

https://orcid.org/0000-0001-6883-5061

Submitted on 05.05.2021 and accepted for publication on 05.07.2021

\section{Introduction}

Mycotic aneurysms are rare but they have a high morbidity and mortality (1). They are due to infection of the vascular wall by bacteria, not by fungi as the term implies (2). Hence the term "mycotic" is misnomer. Commonly non-hemolytic type streptococci, staphylococci, pneumococci and Salmonella spp. cause this infection (3). Melioidosis is very rare to cause mycotic aneurysms (4). The formation of a mycotic aneurysm is initiated by lodging of bacteria on the inner surface of the vessels or the vasa vasorum (5). Thereafter, local inflammation progresses and make the vessels fragile, leading to rupture.

Mycotic aneurysms occur most frequently in the aorta followed by cerebral, superior mesenteric, femoral, splenic, hepatic and brachial arteries (6). Risk factors include hypertension, emotional stress, aging, changed environment, allergy and smoking and diseases such as rheumatic fever (3).

Melioidosis is considered as a very rare disease in most parts of the world. But it is endemic in Southeast Asia and Northern Australia (4). Burkholderia pseudomallei, the organism that melioidosis was reported as the most common causative agent of mycotic aneurysm in NorthEastern Thailand (4). It is a granulomatous infectious disease associated with high mortality and morbidity despite prompt diagnosis and treatment (5). This bacterium is commonly found in soil and water. Infection is primarily via direct contact and inoculation of contaminated water or soil classically in paddy fields (4). The inoculation period of this organism ranged from 2 days to 26 years (4). Apart from a mycotic aneurysm in superficial femoral artery due to mixed bacterial species, there are no reported cases on mycotic aneurysms caused by melioidosis in Sri Lanka (7). We report a case of 66-year-old patient who presented with a ruptured common iliac artery mycotic aneurysm.

\section{Case presentation}

A 66-year-old retired police officer presented with acute onset of dull pain in the right iliac fossa radiating to the upper thigh. Pain was persistent and did not resolve with simple analgesics. He was afebrile and there was no vomiting, anorexia or loss of appetite. His bowel habits were normal. He had no family history of malignancy. There was mild pallor in him and he had a pulse rate of $110 \mathrm{bpm}$. His blood pressure was $100 / 70 \mathrm{mmHg}$. There was a pulsatile tender mass over the right iliac fossa. The dorsalis pedis and posterior tibial pulses on the right side were not felt. An urgent contrast CT angiogram of both lower limbs was done (Figure 1).

CT showed leaking common iliac artery saccular aneurysm on the right side. The patient was prepared for urgent exploratory laparotomy. Meanwhile, subcutaneous morphine $5 \mathrm{mg}$ was given as an initial dose. He was kept fasting and given intravenous normal saline 1 litre over 30 minutes as the blood pressure dropped to $70 / 50 \mathrm{mmHg}$. Furthermore, 2 units of crossed match blood were given perioperatively. The broad spectrum antibiotic, meropenem $1 \mathrm{~g}$ IV three times per day was started after obtaining blood for culture. 
Subsequently damage control surgery was done. There was a leaking right common iliac artery aneurysm and a large hematoma over the right iliac fossa. Proximal aortic control was taken. Aneurysmal sac was opened and debrided. Proximal common iliac artery was ligated. Femoral arteries were explored bilaterally. Femoral to femoral cross over bypass grafting were done with $8 \mathrm{~mm}$ polytetrafluroethylene (PTFE). The distal pulses were felt and confirmed by triphasic Doppler wave study after surgery.
Patient was in intensive care for 13 days postoperatively and had hospital-acquired pneumonia which was successfully treated. No other complications developed. The aneurysmal tissue sample was positive for melioidosis. Blood cultures were negative. 2D Echocardiogram did not show features of bacterial endocarditis. The patient was discharged with oral co-trimoxazole $960 \mathrm{mg}$ and oral doxycycline $100 \mathrm{mg}$ twice daily for three months after continuing ceftazidime $1 \mathrm{~g}$ intravenously three times a day for 3 weeks.

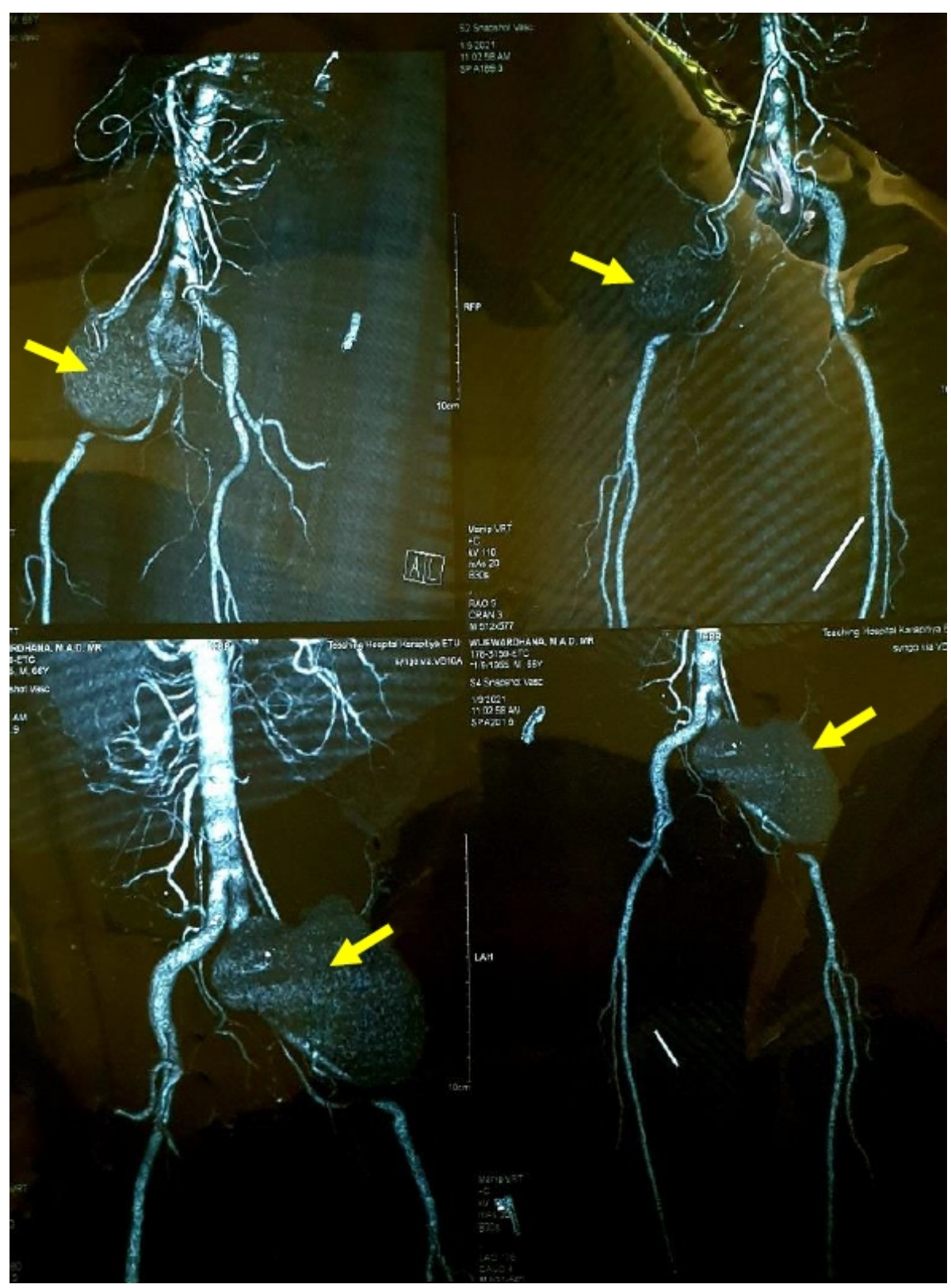

Figure 1: CT angiogram of both lower limbs 


\section{Discussion}

Although mycotic aneurysms are uncommon, they should be considered as important since they tend to grow rapidly and rupture (3). The subsequent surgical procedures are difficult due to inflamed vessels and the prognosis is likely to be poor at the end. Most mycotic aneurysms occur in patients less than 40 years of age in contract to arteriosclerotic aneurysms (3). The bulk of the mycotic aneurysms involved the peripheral arteries and are seven times frequent than aortic mycotic aneurysms (2). Among them, femoral artery aneurysms were the commonest while there were only a few reported cases of common iliac artery aneurysms (2).

In the literature, there are several theories that describe the pathogenesis of mycotic aneurysms. Currently accepted theory is the lodging of bacteria over the intima of vasa vasorum which supply the major vessels. Local ischemia will sets in and it initiates local inflammation and subsequent degeneration of the vessel wall $(3,8)$. Ultimately the vessel wall will be fragile enough to rupture. There are two types of mycotic aneurysms according to the pathogenesis. They are primary and secondary types. Primary mycotic aneurysms are due to direct extension of an adjacent suppurative focus while secondary mycotic aneurysms are due to septic emboli due to endocarditis (9).

There are cases of mycotic aneurysms of aortic arch, subclavian artery and femoral arteries by mixed bacteria. But common iliac mycotic aneurysms have not been documented. Melioidosis is a rare but well documented cause of mycotic aneurysms and is described to occur in around $1 \%$ of affected patients (10). Therefore, mycotic aneurysms are not a very common complication of melioidosis. All the previous cases in the literature were treated by early surgical interventions which was similar to our case. This case illustrates a very rare disease which carries high morbidity and mortality rates. Thus early diagnosis is imperative despite of the rarity.

\section{Conclusions}

Melioidosis mycotic aneurysms are exceedingly rare. But due to the disastrous nature of the infection, early diagnosis and prompt treatment with prolonged antibiotics must be implemented. It is imperative to treat these aneurysms with early surgical intervention. A clinical suspicion of melioidosis mycotic aneurysm is pivotal in Sri Lanka where paddy fields are abundant.

\section{References}

1. Anderson C.B, Mycotic aneurysms. Archives of Surgery. 1974: 109(5): 712-717. doi: 10.1001/archsurg.1974.01360050106022.

2. Sayers R, Davies RSM John AM. Arterial disorders. In: Williams NS, O'Connell PR, McCaskie AW, editors. Bailey \& Love's Short Practice of Surgery. $27^{\text {th }}$ edition. London: Taylor \& Francis; 2018. p.960-961.

3. Nakata Y, Shionoya S Kamiya K. Pathogenesis of mycotic aneurysm. Angiology. 1968; 19(10): 593-601. doi: 10.1177/000331976801901003.

4. Li PH, Chau CH, Wong PC. Melioidosis mycotic aneurysm: An uncommon complication of an uncommon disease. Respiratory Medicine Case Reports. 2015; 14: 4346. doi:10.1016/j.rmcr.2014.12.005.

5. Stengel A, Wolferth CC. Mycotic (bacterial) aneurysms of intravascular origin. Arch. Int. Med. 1923; 31:527-554.

6. Hoover BA II, Lampe WT II. Mycotic aneurysm of the forearm following treated bacterial endocarditis. Angiology. 1965; 16: 203-208.

7. Gooneratne TD, Sriskantha S, Wijeyaratne MA. Case of a mycotic aneurysm of the superficial femoral artery infected by mixed bacterial species. Annals of Vascular Diseases. 2011; 4(4):325-327. doi:10.3400/avd.cr.11.00018.

8. Zak FG, Strauss L, Saphra I. Rupture of diseased large arteries in the course of enterobacterial (Salmonella) infections. New England J. Med. 1958; 258(17): 824-828.

9. Brown SL, Busuttil RW, Baker JD, Machleder HI, Moore WS, Barker WF. Bacteriologic and surgical determinants of survival in patients with mycotic aneurysms. Journal of Vascular Surgery. 1984; 1(4): 541-547. doi: 10.1016/07415214(84)90040-5.

10. Low JG, Quek AM, Sin YK, Ang BS. Mycotic aneurysm due to Burkholderia pseudomallei infection: case reports and literature review. Clin Infect Dis. 2005; 40(1): 193-198. 